\title{
Machine Learning Methods for Content - Classification and Categorization
}

\author{
${ }^{1}$ Prabhat Kubal, ${ }^{2}$ Prof. Surabhi Thorat, ${ }^{3}$ Prof. Swati Maurya
}

${ }^{1}$ Student, Department of Computer Science, S.K.Somaiya College, Somaiya Vidyavihar University, Mumbai, India

${ }^{2,3}$ Professor, Department of Computer Science, S.K.Somaiya College, Somaiya Vidyavihar University, Mumbai, India

\begin{abstract}
Article Info

Volume 7, Issue 6

Page Number : 184-189

Publication Issue :

November-December-2021

Article History

Accepted : 01 Dec 2021

Published : 07 Dec 2021

These days online gatherings and web-based media stages have furnished people with the necessary resources to advance their contemplations and put themselves out there free paying little heed to the kind of language used to communicate those thoughts, in certain examples these internet based remarks contain express language which might hurt the peruser. We likewise evaluate the class irregularity issues related with the dataset by utilizing inspecting procedures and misfortune. Models we applied yield high in general exactness with moderately minimal expense. To diminish the adverse consequence of poisonous remark in everyday life we have endeavored to plan a Toxic Language detector.
\end{abstract}

Keywords : Text Detection, Text Analysis, Content Analysis, Social Media, Education, Toxic Language Classifier

\section{INTRODUCTION}

Content examination is an exploration technique used to distinguish designs in recorded correspondence. To direct substance examination, you deliberately gather information from a bunch of texts, which can be composed, oral, or visual. Individuals have been looking for help from different devices to investigation text-based data so they can recognize harmful articulations from an ocean of data, both effectively, and all the more critically, precisely.
As we as a whole know in this creating universe of online media there, are different effect on human existence, of which some are positive and some are negative. Negative remarks can cause different issues like discouragement, nervousness, fit of anxiety and so on To lessen such things, we are attempting to foster programming.

\section{LITERARY REVIEW}

2.1 UCLA

UCLA Electronic Theses and Dissertations

Utilization of Recurrent Neural Networks in Toxic Comment Classification (Li, Sycuan) 2018 Internet cynicism has consistently been an interesting issue. 
The obscurity and the feeling of distance of individuals' web presence have urged individuals to communicate their thoughts openly. This opportunity can now and then prompt outrageous outtakes on others individuals or the specific points.

Outrageous negativities has at times prevented individuals from putting themselves out there or made them quit any pretense of searching for various conclusions online Issues like this happen practically constantly, across all foundation of conversation, and the modulators of these stages have restricted capacities managing it. Obviously the time, energy and exertion these modulators need to place into controlling this pessimism on their foundation, individuals have been looking for help from different devices to investigate text-based data with the goal that they can distinguish harmful articulations from an ocean of data, productively, and all the more critically, precisely, In this proposal, we will apply word installing strategies and repetitive neural organization to perform text grouping on a multimark text dataset to recognize various types of web poisonousness. Normal language handling with profound Neural Networks is perhaps the most compelling tool that empower scientists to extricate, investigate, and arrange fundamental highlights from text-based data.

\subsection{Random Forest Algorithm Report}

Random forests are built by combining the predictions of several trees, each of which is trained in isolation.

There are three fundamental decisions to be made while developing an arbitrary tree. These are the strategy for parting the leaf's, The kind of indicator to use in each leaf, and the technique for infusing haphazardness into the trees. Indicating a technique for parting leaf's requires choosing the states of applicant parts just as a strategy for assessing the nature of every up-and-comer. To part a leaf, an assortment of competitor parts are produced and a rule is assessed to pick between them. The most widely recognized for indicators in each leaf is to utilize the normal reaction over the preparation focuses which fall in that leaf. Distinctive leaf indicators for relapse and different assignments, however these speculations are past the extent of this paper. We consider just basic averaging indicators here.

\subsection{Toxic Comment Detector Report}

To recognize and characterize poisonous web-based analysis, the cutting edge instruments of information science change crude text into key highlights from which either learning calculations can make expectations for checking hostile discussions. We methodicallly think about 62 classifiers addressing 19 significant algorithmic families in contrast to highlights extricated from the Jigsaw dataset of Wikipedia remarks. We look at the classifiers dependent on measurably huge contrasts in exactness and relative execution time. Among these classifiers for distinguishing harmful remarks, tree-based calculations give the most straightforwardly reasonable standards and rank request the prescient commitment of each component. Among 28 highlights of language structure, feeling, feeling and exception word references, a straightforward terrible word list demonstrates generally prescient of hostile editorial.

\begin{tabular}{|l|c|c|}
\hline \multicolumn{1}{|c|}{ Comment } & \multicolumn{2}{|c|}{ Toxic Rating } \\
\hline $\begin{array}{l}\text { We suck at dealing with abuse and } \\
\text { trolls on the platform and we've } \\
\text { sucked at it for years }\end{array}$ & 0.77 & Toxic \\
\hline $\begin{array}{l}\text { We suck at dealing with abuse and } \\
\text { trolls on the platform but we'll get } \\
\text { better at it. }\end{array}$ & 0.74 & Toxic \\
\hline $\begin{array}{l}\text { We don't suck at dealing with abuse } \\
\text { and trolls on the platform and } \\
\text { we've never sucked at it }\end{array}$ & 0.64 & Unsure \\
\hline $\begin{array}{l}\text { We're not good at dealing with } \\
\text { abuse and trolls on the platform and } \\
\text { we've sucked at it for years }\end{array}$ & 0.61 & Unsure \\
\hline $\begin{array}{l}\text { We're not good at dealing with } \\
\text { abuse and trolls on the platform but } \\
\text { we'll get better at it. }\end{array}$ & 0.35 & Unlikely \\
\hline $\begin{array}{l}\text { We're not good at dealing with } \\
\text { fame and fortune on the platform } \\
\text { but we'll get better at it. }\end{array}$ & 0.06 & Untikely \\
\hline
\end{tabular}

FROM THE ABOVE RESEARCH PAPER, WE HAVE BEEN INSPIRED TO CATEGORIZE THE COMMENT INTO DIFFERENT PARAMETERS SUCH AS INSULT, TOXICITY, ETC. 


\section{PROBLEM DEFINATION}

The information we will zero in on is a public dataset given by the Conversation AI group; an examination start helped to establish by Jigsaw and Google. Jigsaw is an innovation hatchery made by Google, with the essential target to "use innovation to handle the hardest international difficulties, from countering vicious radicalism to ruining on the web restriction to moderating the dangers related with advanced assaults. Jigsaw and Google dispatched Perspective API in February 2017, a free apparatus that uses AI to distinguish harmful remarks. To work on the presentation of the Perspective API, and with the conviction that "communitarian critical thinking yields the best arrangements the Conversation AI group facilitated a "Wikipedia Talk Page Comments clarified with poisonousness reasons" Kaggle contest. We will assemble our profound learning grouping model and screen its exhibition base on the dataset gave in this opposition. Right now, the model utilized by Conversation API performs very well, ready to give a moderately exact poisonous score given text remarks. Notwithstanding, the group referenced that their model actually makes mistakes it can't characterize poisonousness in case the model has not seen the example previously, and it might missarrange texts that share comparable examples as harmful remarks. Intermittent neural organizations' capacity to handle arrangements of archives and break down settings might demonstrate valuable in settling the issues Conversation API right now experiences

\section{METHODOLOGY | APPROACH}

\subsection{What Is NLP?}

NLP utilizes perceptual, conduct and correspondence methods to make it more straightforward for individuals to change their contemplations and activities NLP depends on language handling yet ought not be mistaken for normal language handling, what has a similar abbreviation. For instance, a focal element of NLP is the possibility that an individual is one-sided towards one tangible framework, known as the favored authentic framework or PRS. Specialists can identify this inclination through language Phrases, for example, "I see your point might flag a visual PRS. Or on the other hand "I hear your point" may flag a hear-able PRS. A NLP specialist will recognize an individual's PRS and base their restorative structure around it. The structure could include compatibility building. Data get together, and objective setting with them.

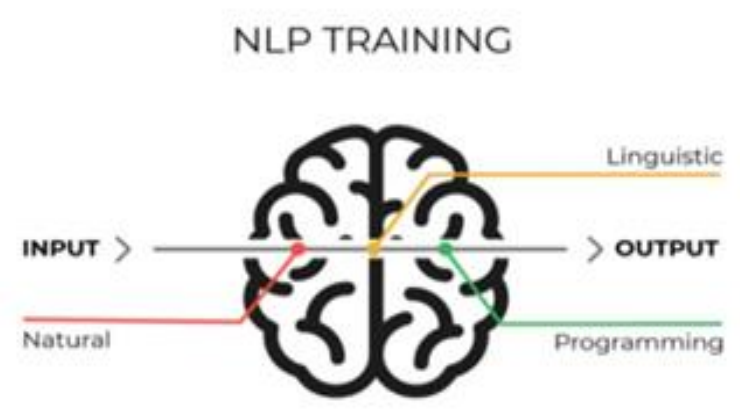

Figure 1 : NLP Training

In this thesis, we will be applying word embedding techniques and recurrent neural network to perform text classification on a multi-label text dataset to identify different forms of internet toxicity

\subsection{Random Forest algorithm}

Random forest is an administered learning calculation which is utilized for both arrangement just as relapse. In any case, notwithstanding, it is mostly utilized for characterization issues. As we realize that a woodland is comprised of trees and more trees implies more vigorous backwoods. Additionally, Random forest calculation makes choice trees on information tests and afterward gets the forecast from every one of them lastly chooses the best arrangement through casting a ballot. It is an outfit strategy which is superior to a solitary choice tree since it decreases the over-fitting by averaging the outcome. 


\section{Working of Random Forest Algorithm}

We can understand the working of Random Forest algorithm with the help of following steps-

Step 1 - First, start with the selection of random samples from a given dataset.

Step 2 - Next, this algorithm will construct a decision tree for every sample. Then it will get the prediction result from every decision tree.

Step 3 - In this step, voting will be performed for every predicted result.

Step 4 - At last, select the most voted prediction result as the final prediction result.

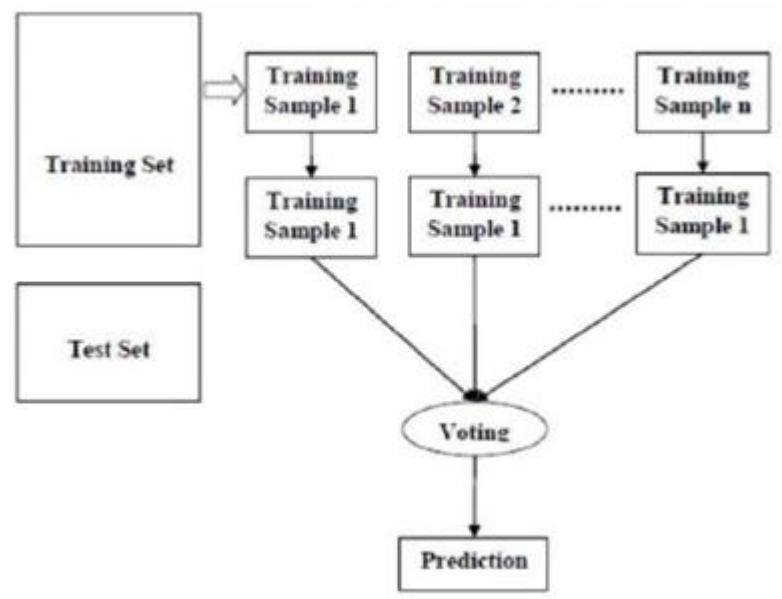

Figure 2 : Working of Random Forest Algorithm

These labels were summarized into a total of six classes:

Toxic: A general classification of the toxicity of the comments.

Severe Toxic: Extreme toxicity.

Obscene: Indecent language.

Threat: Statements with the intention to perform hostile action.

Insult: Disrespect or verbal abuse.

Identity Hate: Sexism, racism, homophobic, etc.

4.3 Some of the important concepts used are:

Counter vectorization

The Count Vectorizer provides a simple way to both tokenize a collection of text documents and build a vocabulary of known words, but also to encode new documents using that vocabulary.

\section{Tdif vectorization}

It can be used as input to estimator. Vocabulary, Is a dictionary that converts each token (word) to feature index in the matrix, each unique token gets a feature index.

Pickles

"Pickling" is the process whereby a Python object hierarchy is converted into a byte stream, and "unpicking" is the inverse operation, whereby a byte stream is converted back into an object hierarchy.

\section{Tokenization}

In Python tokenization basically refers to splitting up a larger body of text into smaller lines, words or even creating words for a non-English language.

\subsection{Basic Data Flow Diagram}

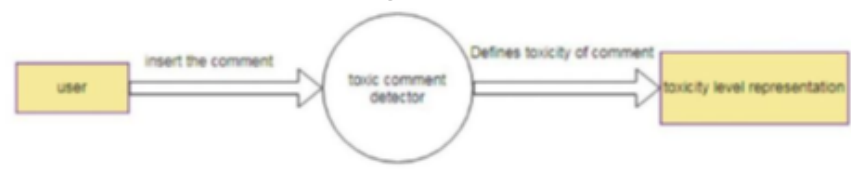

Figure 3 : Basic Data Flow Diagram

\section{RESULTS}
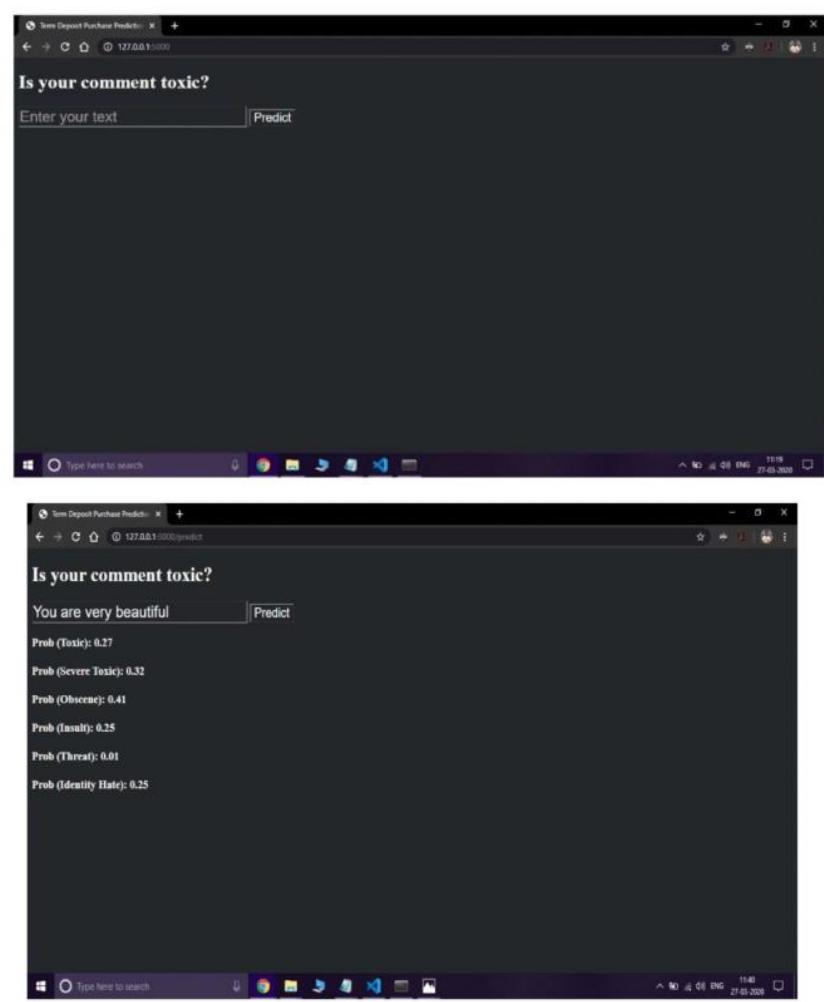
It includes all positive words in sentence. Hence all the categories in the output are minimal

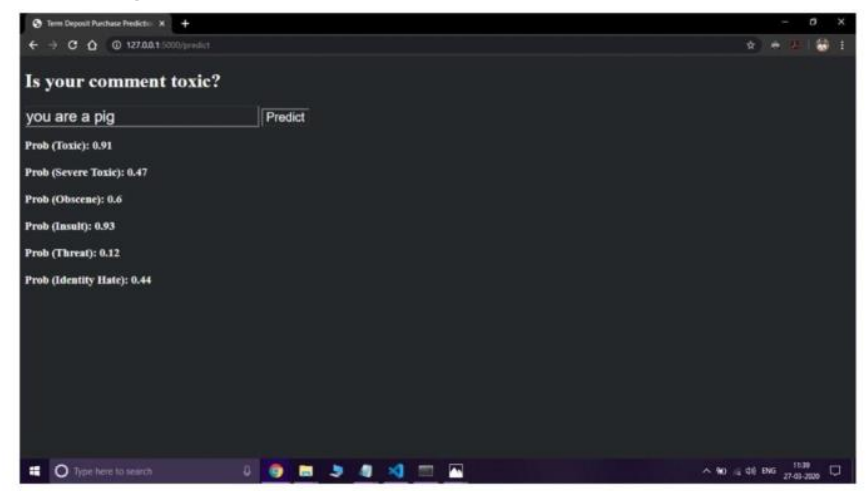

In this case word 'pig' is categorized in insult section. Hence the software has displayed the probability of insult parameter as maximum as compared to other parameters.

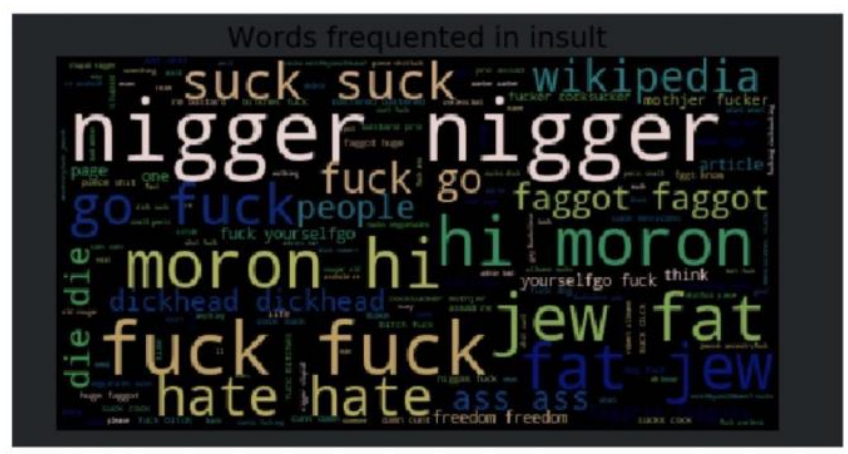

These are some other examples of toxicity or insult.

\section{CONCLUSION}

Toxic Language Detector can additionally be utilized for identifying remarks remark on applications which are like WhatsApp, Facebook, Instagram, and so on When contrasted with other different calculations irregular woodland calculation has been demonstrated extensively compelling. It can been seen through the accompanying chart.

These observations likewise imply that further enhancements can be made to work on our present model, which doesn't restrict to an alternate generally speaking organization structure. We can likewise play out extra hyperparameter tuning on our model, which will most certainly demonstrate valuable. All things considered, the model's capacity to handle setting of words demonstrates productive in recognizing poisonous texts from a huge example.

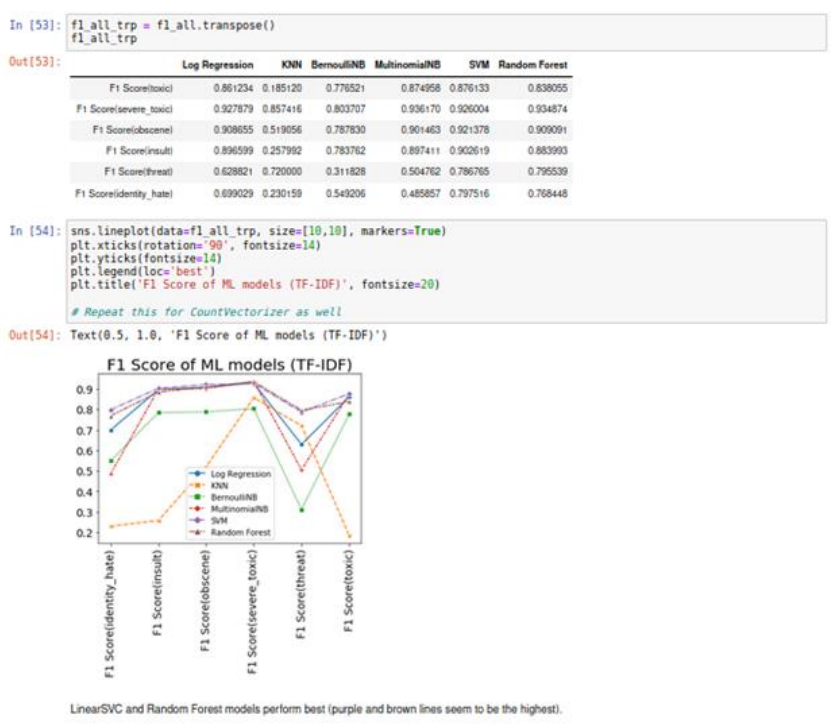

\section{ACKNOWLEDGEMENT}

I sincerely wish to thank the project guide Prof. Surabhi Thorat for her encouraging and inspiring guidance helped me to make my project and research a success. The project guide made endure with her expert guidance, kind advice and timely motivation which helped me to determine my project.

I would like to thank my H.O.D Prof. Swati Maurya for all the support we needed from her for our project without her kind and keen co-operation our project would have been stifled to standstill.

\section{REFERENCES}

[1]. Maslej Kresnakova, Viera \& Sarnovsky, Martin \& Butka, Peter \& Machova, Kristina. (2020). Comparison of Deep Learning Models and Various Text Pre-Processing Techniques for the Toxic Comments Classification. Applied Sciences. 10. 8631. 10.3390/app10238631.

[2]. Maslej Krešňáková, Viera \& Sarnovsky, Martin \& Butka, Peter \& Machova, Kristina. (2020). 
Comparison of Deep Learning Models and Various Text Pre-Processing Techniques for the Toxic Comments Classification. Applied Sciences. 10. 8631. 10.3390/app10238631.

[3]. Pavel, Monirul. (2020). Toxic Comment Classification Implementing Natural Language Processing and Convolutional Neural Networks With Word Embedding Technique.

[4]. Gupta, Akash \& Nayyar, Anand \& Arora, Simrann \& Jain, Rachna. (2021). Detection and Classification of Toxic Comments by Using LSTM and Bi-LSTM Approach. 10.1007/978981-16-3660-8_10.

[5]. Georgakopoulos, Spiros \& Tasoulis, Sotiris \& Vrahatis, Aristidis \& Plagianakos, Vassilis. (2020). Convolutional Neural Networks for Twitter Text Toxicity Analysis. 10.1007/978-3030-16841-4_38.

[6]. van Aken, Betty \& Risch, Julian \& Krestel, Ralf \& Löser, Alexander. (2018). Challenges for Toxic Comment Classification: An In-Depth Error Analysis.

[7]. Androcec, Darko. (2020). Machine learning methods for toxic comment classification: a systematic review. Acta Universitatis Sapientiae, Informatica. 12 205-216. 10.2478/ausi-2020-0012.

[8]. Pavel, Monirul \& Razzak, Razia \& Sengupta, Katha \& Niloy, Dilshad Kabir \& Muquith, Munim \& Tan, Siok. (2021). Toxic Comment Classification Implementing CNN Combining Word Embedding Technique. 10.1007/978-98133-4305-4_65.

[9]. Rani, Pooja \& Panichella, Sebastiano \& Leuenberger, Manuel \& Di Sorbo, Andrea \& Nierstrasz, Oscar. (2021). How to Identify Class Comment Types? A Multi-language Approach for Class Comments Classification.

\section{Cite this article as :}

Prabhat Kubal, Prof. Surabhi Thorat, Prof. Swati Maurya, "Machine Learning Methods for Content Classification and Categorization", International Journal of Scientific Research in Computer Science, Engineering and Information Technology (IJSRCSEIT), ISSN : 2456-3307, Volume 7 Issue 6, pp. 184-189, November-December 2021. Available at doi : https://doi.org/10.32628/CSEIT217648 Journal URL : https://ijsrcseit.com/CSEIT217648 